\title{
Extracellular vesicle associated and soluble immune marker profiles of psychoneurological symptom clusters in men with prostate cancer: an exploratory study
}

\author{
Dilorom Sass (iD ${ }^{1,2}$, Leorey Saligan (iD ${ }^{1 凶}$, Wendy Fitzgerald ${ }^{3}$, Ann M. Berger ${ }^{2}$, Isaias Torres ${ }^{1}$, Jennifer J. Barb (D) ${ }^{4}$, Kevin Kupzyk ${ }^{2}$ and \\ Leonid Margolis ${ }^{3}$
}

This is a U.S. government work and not under copyright protection in the U.S.; foreign copyright protection may apply 2021

\begin{abstract}
Psychoneurological symptom clusters are co-occurring and interrelated physiological symptoms that may include cancer-related fatigue, pain, depressive symptoms, cognitive disturbances, and sleep disturbances. These symptoms are hypothesized to share a common systemic proinflammatory etiology. Thus, an investigation of systemic immune biomarkers is an important approach to test this hypothesis. Here, we investigated the associations between extracellular vesicle (EV)-associated and soluble cytokines with immune markers and symptom clusters in men with non-metastatic prostate cancer. This observational study included 40 men with non-metastatic prostate cancer at the start (T1) of external beam radiation therapy (EBRT) and 3 months post treatment (T2), as well as 20 men with non-metastatic prostate cancer on active surveillance (AS) seen at one time point. Collected questionnaires assessed patient-reported fatigue, sleep disturbances, depressive symptoms, and cognitive fatigue. In total, 45 soluble and EVassociated biomarkers in plasma were determined by multiplex assays. Principal component analysis (PCA) was used to identify psychoneurological symptom clusters for each study group and their time points. Bivariate correlation analysis was run for each identified PCA cluster with the concentrations of EV-associated and soluble cytokines and immune markers. Both EV-associated and soluble forms of RANTES significantly correlated with the symptom cluster for EBRT at T1, whereas, at T2, soluble IFNa2, IL-9, and IL17 correlated with the corresponding symptom cluster. For the AS group, soluble survivin correlated with psychoneurological symptoms. Linking specific inflammatory cytokines with psychoneurological symptom clusters in men receiving prostate cancer treatment can enhance understanding of the underlying mechanisms of this phenomenon and aid in developing targeted interventions.
\end{abstract}

Translational Psychiatry (2021)11:440; https://doi.org/10.1038/s41398-021-01554-w

\section{INTRODUCTION}

Cancer survivors often face many co-occurring, psychoneurological symptoms such as cancer-related fatigue, pain, depression, sleep disturbances, and cognitive impairments [1-3] that can lead to poor health-related quality of life [4]. These interrelated symptoms form clusters that seem to be related to psychological and/or neurological dysfunction [5] in patients with cancer.

Multiple studies connected psychoneurological symptom clusters to inflammation, activation of sympathetic nervous system, and activation of hypothalamic-pituitary-adrenal (HPA) axis) [2]. Previously identified cytokine gene polymorphisms in patients with higher symptom burden include C-reactive protein (CRP), interleukin 4 (IL-4), IL-6, IL-7, and soluble tumor necrosis factor receptor 1 (sTNF-R1) [2]. It has been hypothesized that peripheral inflammation affects these symptoms through the expression of cytokines within the brain triggering sickness-like behavior, including fatigue and depression $[6,7]$.

In patients with cancer treated with cytokines (e.g. IL-2, IL-2 + IFNa, and IFNa), $50 \%$ of subjects developed mild depressive symptomology and $22 \%$ developed moderate to marked depressive symptomology. Moreover, cytokine administration led to the development of neurovegetative symptoms (decreased sleep and appetite), cognitive disturbances, and emotional affective symptoms (sadness, inner tension) [8]. In another study, in patients with non-small lung cancer treated with epidermal growth factor receptor tyrosine kinase inhibitor, the circulating levels of proinflammatory chemokine, RANTES, was significantly associated with increased severity of general fatigue [9]. In contrast, in patients with psoriasis, injections of TNF-blocking agent, Entracept, resulted in reduced fatigue and depressive symptoms [10]. These studies affirm that inflammatory cytokines play a role in expression of psychoneurological symptoms.

Clinically, it is challenging to draw clear connections between peripheral cytokines and the brain; thus, peripheral markers that are able to cross blood-brain barrier can be informative in relation to behavioral outcomes. Extracellular vesicles (EVs) could serve as markers since it was shown that they can cross the blood-brain barrier and carry inflammatory and neurogenerative molecules from periphery to the brain and vice versa [11].

\footnotetext{
${ }^{1}$ National Institute of Nursing Research, National Institutes of Health, Bethesda, MD, USA. ${ }^{2}$ University of Nebraska Medical Center, Omaha 68105 NE, USA. ${ }^{3}$ Section on Intercellular Interactions, Eunice Kennedy-Shriver National Institute of Child Health and Human Development, National Institutes of Health, Bethesda, MD, USA. ${ }^{4}$ Clinical Center, National Institutes of Health, Bethesda, MD, USA. ${ }^{凶}$ email: saliganl@mail.nih.gov
} 


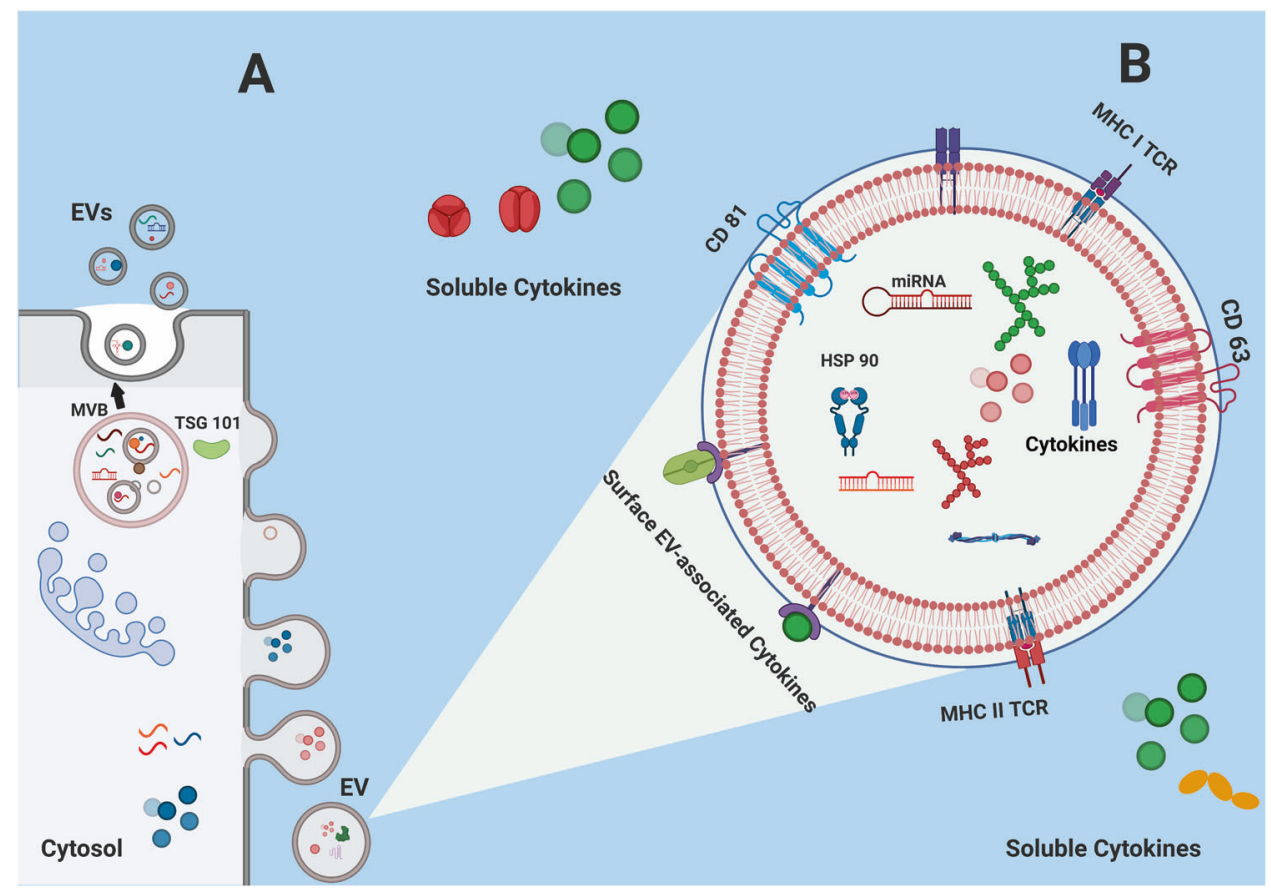

Fig. 1 Illustration of extracellular vesicles (EV). A. Release of EVs from multivesicular body (MVB); B EV with common genetic components and proteins including cytokines [12, 13].

EVs (30-1000 $\mathrm{nm}$ in diameter) produced by cells of many types carry tetraspanins (CD9, CD63, CD81, CD82), heat-shock proteins (HSP70, HSP90), cytokines, miRNAs, MHC class I and MHC class II, and many proteins $[12,13]$. Figure $1 \mathrm{~A}$ illustrates release of EVs from a multivesicular body and Fig. 1B illustrates an enlarged image of an EV with previously described common genetic and protein markers $[12,13]$.

Earlier, it was demonstrated that cytokines are present in EVassociated and free (soluble) forms in a number of in vitro, ex vivo, and in vivo systems and both forms of the cytokines are biologically active [14]. Investigation of EV-associated cytokines relations to the psychoneurological symptoms may reveal their role as behavioral mediators. To our knowledge, such an investigation has not been previously undertaken. Therefore, the aim of our exploratory study was to investigate the associations of EV-associated and soluble cytokines, chemokines, and heat-shock proteins in two cohorts: men with non-metastatic prostate cancer treated with external beam radiation therapy (EBRT) and those on active surveillance (AS) experiencing psychoneurological symptom clusters.

\section{SUBJECTS AND METHODS \\ Subjects}

The study was approved by the Institutional Review Board (IRB) of the National Institutes of Health (NIH) (NCT00852111). Study participants were recruited from the NIH Clinical Center between 2009 and 2014. Informed consents were obtained prior to study procedures. Men with nonmetastatic prostate cancer, who were scheduled to receive EBRT $(n=$ 40), were recruited. Study samples and instruments were collected at two time points: at the start of EBRT (T1) and 3 months after completion of EBRT (T2). The inclusion criteria for the EBRT cohort were: $\geq 18$ years of age, scheduled to receive EBRT with or without concomitant androgen deprivation therapy, and the ability to provide written informed consent. Men with prostate cancer on AS $(n=20)$ were also recruited and seen at the NIH Clinical Center in one outpatient visit. Participants on AS were included if they had clinically localized prostate cancer, had been placed on $\mathrm{AS}$, and did not receive previous or current treatment for prostate cancer. A convenience sample size was selected of all participants with available blood collection and complete clinical and behavioral outcomes.

\section{Instruments}

The Functional Assessment Cancer Therapy-Fatigue (FACT-F). Fatigue levels were assessed using the FACT-F, a validated fatigue assessment tool with a 13-item fatigue scale (Pearson $r=0.87$, Cronbach alpha $=0.93-0.95)[15,16]$ using a Likert scale rated from "not at all $=0$ " to "very much $=4$ ". The total scores range from 0 to 52 . The lower the score, the greater the intensity and interference of fatigue on daily life.

Hamilton Depression Scale (HAM-D). HAM-D is a 21-item scale to assess the severity of depressive symptoms. This questionnaire is reported to have good internal reliability (Cronbach alpha 0.46-0.97), inter-rater reliability (Pearson $r=0.82-0.98)$, and retest reliability $(r=0.81-0.98)$ [17]. In the clinic, HAM-D scores of 0-7 indicates no depression, 8-13 mild depression, 14-18 moderate depression, 19-22 severe depression, and $\geq 23$ very severe depression [18]. For the purposes of this study, continuous values were used with higher values indicating greater severity [18].

Sleep disturbances. The Patient Reported Outcomes Measurement Information System (PROMIS) Sleep Disturbances and Sleep Related Impairment (SD and SRI) is an eight-item questionnaire using a Likert scale rated from "not at all" to "very much" with previously established construct validity and reliability coefficient of 0.90 [19]. Like other PROMIS measures, the PROMIS Sleep Disturbances and Sleep Related Impairment raw scores were converted to $T$-scores to distinguish the responses from the US general population ( $T$-score of $>50 \pm 10 \mathrm{SD}$ ). These two questionnaires assess the severity of sleep disturbances and sleep-related impairment $[19,20]$.

\section{Revised Piper Fatigue Scale (rPFS) (cognitive/mood fatigue subscale)}

The rPFS is a 22-item questionnaire that includes four fatigue dimensions: behavioral/severity (six items), sensory (five items), affective meaning (five items), and cognitive/mood (six items). Only the cognitive/mood fatigue subscale was used in this study, with higher scores indicating greater cognitive fatigue severity. Cronbach's alpha for rPFS subscales have been reported as $0.89-0.97$ [21].

\section{Blood collection}

Peripheral blood was drawn by the NIH Clinical Center staff and collected using ethylenediaminetetraacetic acid (EDTA) tubes. Blood was processed for plasma following standard NINR procedures and plasma was stored 
at $-80{ }^{\circ} \mathrm{C}$ until analysis. The primary author ran all the samples together; hence, was blinded to individual subject symptom scores during the experiment (multiple assay).

\section{EV isolation}

EVs were isolated from thawed plasma using ExoQuick ${ }^{\mathrm{TM}}$ (SystemBio, Palo Alto, CA) following the manufacturer's instructions. Each plasma sample was centrifuged twice at $3000 \mathrm{~g} \times 15 \mathrm{~min}$ to deplete the sample of platelets and arrive at platelet poor plasma (PPP). Platelet depleted $250 \mu \mathrm{l}$ of plasma was incubated with $63 \mu \mathrm{l}$ of Exoquick ${ }^{\mathrm{TM}}$ for $30 \mathrm{~min}$ at $4{ }^{\circ} \mathrm{C}$. The sample was centrifuged at $1500 \mathrm{~g} \times 30 \mathrm{~min}$ at $4^{\circ} \mathrm{C}$, the $\mathrm{EV}$-free supernatant was collected, and the EV pellet was resuspended in $250 \mu \mathrm{l}$ of sterile PBS. Multiplexed bead-based assays were run the same day as the sample preparation. EVs were characterized for size, morphology, and presence of $\mathrm{CD}$ markers, as previously described [22].

\section{Cytokine and heat-shock protein measurements}

Concentrations of various inflammatory cytokines and stress-related proteins were measured from total EVs isolated from plasma and were assessed using multiplexed bead-based assays (Luminex). Two panels of markers included IL-1a, IL-1 $\beta$, IL-2, IL-4, IL-6, IL-7, IL-8, IL-10, IL-12p70, IL-13, IL-15, IL-16, IL-17, IL-18, IL-21, IL-22, IL-33, Calgranulin A (S100A8), Eotaxin (CCL11), granulocyte-macrophage colony-stimulating factor (GM-CSF), growth-regulated alpha (GRO- $\alpha$ or CXCL1), interferon- $\gamma$ (IFN- $\gamma$ ), interferon$\gamma$-induced protein (IP-10 or CXCL10), interferon-inducible $\mathrm{T}$ cell alpha chemoattractant (ITAC or CXCL11), macrophage colony-stimulating factor (M-CSF), monocyte chemoattractant protein-1 (MCP-1 or CCL2), monokine induced by IFN- $\gamma$ (MIG or CXCL9), macrophage inflammatory protein-1a (MIP-1a or CCL3), MIP-1 $\beta$ (CCL4), MIP-3a (CCL20), regulated on activation normally $\mathrm{T}$ cell expressed and secreted (RANTES or CCL5), transforming growth factor- $\beta$ (TGF- $\beta$ ), and tumor necrosis factor- $a$ (TNF- $a$ ), IL-3, IL-6 receptor (R), IL-9, brain-derived neurotrophic factor (BDNF), TNF-related apoptosis-inducing ligand (TRAIL), C-reactive protein (CRP), stromal derived factor (SDF), survivin, and interferon alpha 2 (IFNa2), heat-shock protein (HSP) 27, HSP70, HSP90.

Magnetic beads with distinct spectral signatures were coupled with protein-specific capture antibodies to allow measurement of many different proteins in a single low-volume sample. Catalog numbers and manufacturers for capture and detection antibodies are provided in Supplementary Table 1. Standards (unlysed or lysed with $0.1 \%$ Triton X100 ), intact and lysed EVs, and EV-free supernatants were diluted in assay buffer and then incubated with bead mixes overnight at $4{ }^{\circ} \mathrm{C}$. The next day plates were washed two times and incubated with polyclonal antibodies for $1 \mathrm{~h}$. After two washes, plates were incubated with streptavidinphycoerythrin $(16 \mu \mathrm{g} / \mathrm{ml})$ in PBS (Thermo Fisher). After final washes, beads were resuspended in PBS and cytokines were read on a Luminex 200 System and analyzed with Bioplex Manager software (BioRad). The protocols were previously tested and published [14, 22].

Final concentrations of soluble analytes measured in EV-free supernatants were adjusted to account for dilution by ExoQuick ${ }^{\mathrm{TM}}$ reagent. Surface EV protein was reported by measurement on intact EVs, and internal EV protein was calculated from EV lysed concentrations (lysed minus surface equals internal). Total EV-associated cytokines (surface plus internal) were used for analysis in this study. Lower limits of detection (LLOD) and standard curves were determined using 5P logistic regression. Data on LLOD for each analyte and descriptive statistics for EV concentrations for EBRT cohort at T1 and T2 has been previously published [22]. Data on AS group's EV concentrations (mean and standard deviation) are included in Supplementary Material Table 2.

\section{Statistical analyses}

Descriptive statistics characterized the demographic, clinical, and symptom attributes of the study participants. Data were evaluated for Gaussian distribution by calculating skewness and kurtosis statistics (Supplementary Material Table 3). Two-sided Mann-Whitney $U$ test was used to evaluate group differences between EBRT and AS cohorts for non-normally distributed variables and two-sided independent $T$-test was used to evaluate group differences for normally distributed variables. Two-sided paired $T$-test was used to evaluate differences between EBRT-T1 and EBRTT2 for normally distributed variables and two-sided Wilcoxon signed-rank test was used to evaluate differences between EBRT-T1 and EBRT-T2 for non-normally distributed variables. Pearson chi square test was used for categorical variables. Due to non-normal distributions of the variables, cytokine concentrations were logarithm base ten transformed (Supplementary Table 4). The following symptoms: fatigue, sleep-related impairment, sleep-related disturbances, depression, and cognitive fatigue for each cohort were entered into SPSS to calculate symptoms clusters using principal components analysis (PCA). The symptom clusters identified by PCA were then correlated with the concentrations of logarithm base ten transformed concentrations of the 45 biomarkers using Pearson correlation tests. Assumptions met for PCA analysis are presented in Fig. 2 description. All statistically significant values are indicated by $p<0.05$. IBM SPSS Grad pack 24.0 was used to conduct all statistical analyses. JMP ${ }^{T M}$ Statistical Discovery Software (SAS Headquarters, Cary, NC) was used for hierarchical clustering using the Ward distance metric for both the EV and soluble log ten concentrations. Due to small sample size and explorative nature of the analysis, multiple comparison corrections were not performed. Data are

\begin{tabular}{|c|c|c|}
\hline \multicolumn{3}{|c|}{ Component Matrix EBRT- T1 $a$} \\
\hline & \multicolumn{2}{|c|}{ Component } \\
\hline & 1 & 2 \\
\hline Fatigue & -.873 & \\
\hline Sleep Related Impairment & .926 & \\
\hline Sleep Disturbances & .830 & \\
\hline Cognitive fatigue & 858 & \\
\hline Depressive Symptoms & & .993 \\
\hline
\end{tabular}

\begin{tabular}{|c|c|}
\hline \multicolumn{2}{|c|}{ Component Matrix EBRT- T2 } \\
\hline & Component \\
\hline Fatigue & -892 \\
\hline Sleep Related Impairement & .899 \\
\hline Sleep Disturbances & 866 \\
\hline Cogntive Fatigue & 869 \\
\hline Depressive Symptoms & .425 \\
\hline
\end{tabular}
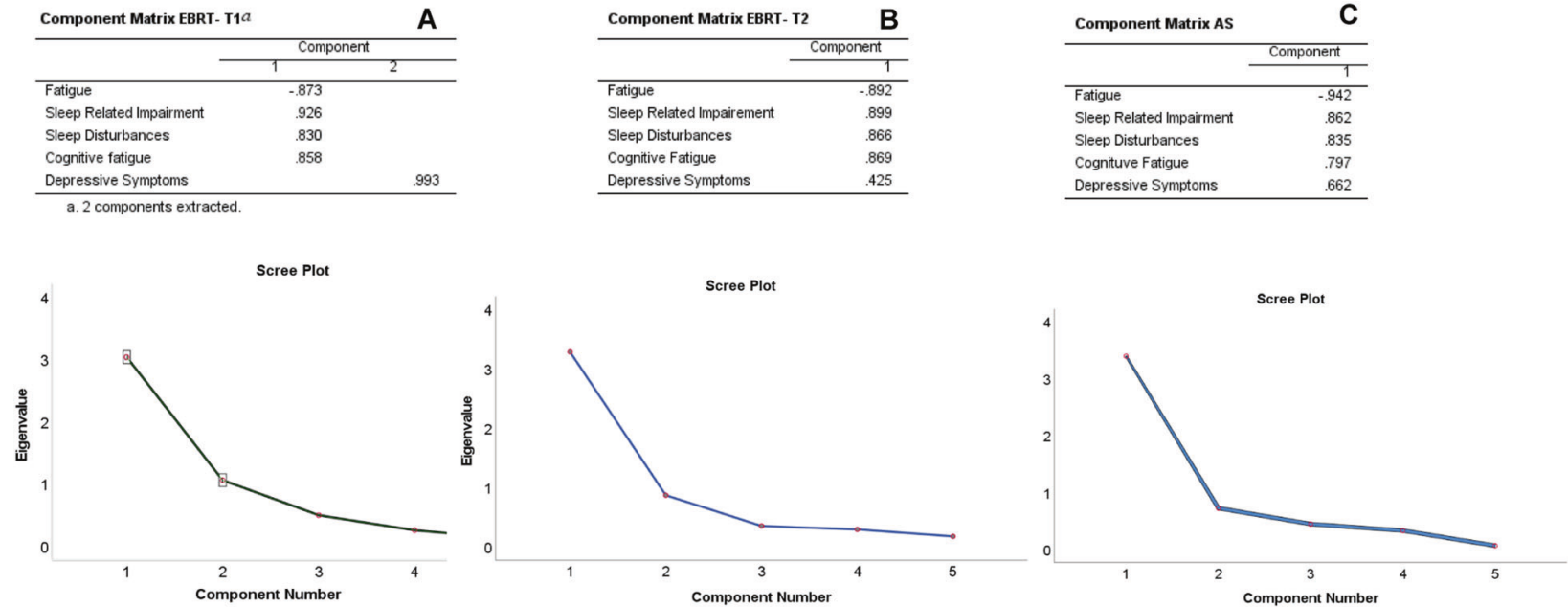

Fig. 2 Principal component analysis results. A Two components were extracted, component 1 had an initial eigenvalue of 3.05 with $60.9 \%$ of variance and component 2 had an initial eigenvalue of 1.01 with $21 \%$ of variance demonstrated by scree plot. Component 2 did not meet clinical definition for symptom cluster [25]; thus, only component 1 is included. Assumptions for PCA met: KMO for sampling adequacy (0.676) and Bartlett's test Chi square for suitability for data reduction $(92.8, p<0.0001)$. B One component was identified for EBRT-T2 cluster with an initial eigenvalue of 3.3 and $65.8 \%$ of total variance. The corresponding scree plot illustrates the component. Assumptions met included KMO for sampling adequacy $(0.82)$ and Bartlett's test Chi square for suitability for data reduction $(102.9, p<0.0001)$. C One component was also identified for AS cluster with initial eigenvalue of 3.4 and $68 \%$ of variance. Corresponding scree plot illustrates the component. Assumptions for testing included KMO for sampling adequacy (0.701) and Bartlett's test Chi square for suitability for data reduction (58.9, $p<0.0001)$. 
presented with mean as measure of center values and standard deviation as measure of estimate of variation.

\section{Missing data}

Five patients in the EBRT group did not complete the sleep disturbances and sleep-related impairment forms at $\mathrm{T} 1$ and one participant did not complete the rPFS mood/cognitive subscale questionnaire at T2. The final data were calculated on the remaining 35 individuals for sleep disturbances and sleep-related impairment questionnaires and 39 individuals for the rPFS mood/cognitive subscale. Two responses $(1,2)$ were marked on the SRI form by one of the participants. Following the PROMIS SRI scoring manual, a data specialist picked one of the two responses by flipping a coin and the selected response was entered into the dataset.

\section{RESULTS}

Clinical and demographic attributes of participants in each group are presented in Table 1. AS men had significantly lower T-stage levels, lower Gleason scores and higher levels of red blood cell (RBC) counts than EBRT men $(p<0.05)$. RBC, hemoglobin, and prostate-specific antigen (PSA) were significantly lower at EBRT-T2 compared to EBRT-T1 $(p<0.001)$.

\section{Symptom clusters}

Principal component analysis was performed on raw symptom scores for each cohort at each time point. Assumptions for sampling adequacy and suitability for data reduction were demonstrated with Kaiser-Meyer-Olkin (KMO) measures and Bartlett's test $[23,24]$. The psychoneurological symptom cluster in EBRT-T1 included all symptoms (CRF, sleep disturbance/sleep impairment, cognitive fatigue). Depressive symptoms (assessed by HAM-D) formed a smaller independent component (Fig. 2) and did not meet clinical criteria for symptom clusters previously defined to include three or more concurrent symptoms [25]. Therefore, it was not counted as part of the cluster. Symptom clusters for EBRTT2 and AS included all four symptoms. Component matrix and scree plots for each symptom cluster are presented in Fig. 2A-C.

\section{EV- associated and soluble markers}

Hierarchical clustering analysis was used to visualize average marker concentrations in soluble and EV-associated fractions for all three groups (EBRT-T1, EBRT-T2, and AS) (Fig. 3). The hierarchical clustering dendrogram was colored at the level of five separate dendrogram breaks to indicate those markers that were expressed similarly across participant groups. As shown in Fig. 3, the first break in the dendrogram (labeled as the teal and orange dendrograms) represent markers that are more expressed in EVs vs. soluble in the EBRT-T1\&2 groups. These markers include IL- $1 \mathrm{~b}$, M-CSF, MIP-3a, IL-17, ITAC, GRO- $\alpha$, TGF- $\beta$, IL-4, IL-3, IL-9, IL-13, CRP, and IL-33.

\section{Correlation of marker concentrations with psychoneurological symptom clusters}

We investigated the correlations of the concentrations of EVassociated and soluble markers with the identified symptom clusters. Each composite symptom score was correlated with the concentrations of the 45 markers in EV-associated and soluble fractions (Table 2). High component scores are indicative of worse symptoms and positive relationships signify that higher biomarker concentrations are associated with worse symptom scores. Negative correlations indicate that higher cytokine/protein concentrations are associated with lower symptom severity.

For the EBRT-T1 psychoneurological symptom cluster, only RANTES had significant positive correlation, indicating worsening symptoms. No significant correlations were found between EV-associated markers and EBRT-T2 psychoneurological symptom cluster. However, there was positive correlation between soluble IFNa2, IL-9, and IL-17 and this symptom cluster.
AS psychoneurological symptom cluster correlated positively only with soluble survivin.

\section{DISCUSSION}

We investigated the associations of EV and soluble immune markers with psychoneurological symptom clusters in men with prostate cancer. We found that: (i) symptoms cluster profiles differed at various cancer treatment trajectories (among AS cohort, before EBRT, and at 3 months post EBRT); (ii) EVassociated and soluble markers correlated with individual symptom clusters (composite scores).

Upregulation of chemokines and cytokines have been previously reported to be associated with worsening fatigue [26], depressive symptoms [27], and cognitive disturbances [28] in patients with cancer. We hypothesized that the proinflammatory immune markers reflect worsening psychoneurological state in patients with prostate cancer.

Our study revealed that at the start of EBRT, soluble and EVassociated RANTES increased with worsening symptoms within EBRT-T1 symptom cluster. RANTES is a member of the chemokine family predominantly produced by CD8 T cells and associated with various inflammatory disorders [29]. An earlier study reported that elevated expression of RANTES correlated with severe general fatigue in patients with lung cancer after tyrosine kinase inhibitor treatment [9]. Furthermore, RANTES was reported to be elevated in men and women with different levels of depression severity [30].

At T2, we found that several soluble markers (IFNa2, IL-9, and IL17) were associated with worse symptoms within EBRT-T2 psychoneurological symptom cluster. All three cytokines share a common signaling pathway through the NFKB complex: IFNa2 increases NFKB activity through IFN-induced genes [31], IL-17 leads to the activation of NFKB [32], and NFkB has been implicated in the expression of IL-9 [33]. Increase in NFKB transcription factors has been previously linked to persistent fatigue in patients with breast cancer [34], and linked to sleep disturbances in healthy adults [35] as well as in patients with long-standing depressive symptoms [36]. This suggests that an increase in proinflammatory cytokines may be associated with the neuropsychological symptom cluster 3 months after treatment completion.

Several publications reported on the association of individual cytokines roles in various psychoneurological symptoms. It was established that cytokine therapy with IFNa is associated with the development of depressive symptoms in patients with malignant melanoma [37]. Furthermore, it was found IFNa therapy for malignant melanoma led to symptoms of fatigue, loss of concentration, gastrointestinal symptoms, and tension/irritability in addition to depressed mood [37]. IFNa treatment in hepatitis C correlated with fatigue, depressed mood, and anxiety ratings [38]. In agreement of these studies, we found that at EBRT-T2, depressive symptoms interrelated with remaining psychoneurological symptoms in PCA and significantly correlated with IFNa2.

Another cytokine that had positive correlation with EBRT-T2 symptom cluster was IL-9. It is a pleiotropic cytokine known to have a dual immunomodulating effect, which largely depends on what subset of Th cells are responsible for its production [39]. Our finding is consistent with a previously reported correlation of IL-9 concentration with worsening fatigue in men with prostate cancer after radiation [26] and chronic fatigue in patients with nonHodgkin's lymphoma [40].

Also, we found that an increase in IL-17 levels correlated with worsening psychoneurological symptoms 3 months after completing radiation therapy. IL-17 is part of six structurally related IL-17 family cytokines known to induce a proinflammatory response in cancer and autoimmune disease [41]. While our study is the first to correlate IL-17 to a psychoneurological symptom cluster, previous reports have shown increased levels of IL-17 to be associated with 
Table 1. Clinical, demographic, and symptoms characteristics of study participants.

\begin{tabular}{|c|c|c|c|c|c|}
\hline & EBRT-T1 $(n=40)$ & EBRT-T2 $(n=40)$ & AS $(n=20)$ & $p$ (EBRT-T1 vs. AS) & $p$ (EBRT-T1 vs. EBRT-T2) \\
\hline Age (years) & $66.9(7.3)$ & & $65.5(9.11)$ & 0.531 & \\
\hline BMI & $29.7(4.5)$ & & $28.18(4.9)$ & 0.214 & \\
\hline Radiation dose & & $7572(276.2)$ & & & \\
\hline Race/Ethnicity & & & & 0.465 & \\
\hline White & $67.5 \%$ & & $85.0 \%$ & & \\
\hline Black/African American & $25.0 \%$ & & $10.0 \%$ & & \\
\hline$T$-stage & & & & $0.008^{*}$ & \\
\hline TO & & & $20.0 \%$ & & \\
\hline T1c & $32.5 \%$ & & $60.0 \%$ & & \\
\hline T2a & $40.0 \%$ & & $20.0 \%$ & & \\
\hline Gleason Scores & & & & $0.000^{*}$ & \\
\hline $3+3=6$ & $7.5 \%$ & & $80.0 \%$ & & \\
\hline $3+4=7$ & $32.5 \%$ & & $15.0 \%$ & & \\
\hline $4+3=7$ & $12.5 \%$ & & $5.0 \%$ & & \\
\hline $3+5=8$ & $2.5 \%$ & & & & \\
\hline $4+4=8$ & $12.0 \%$ & & & & \\
\hline $5+4=9$ & $5.0 \%$ & & & & \\
\hline $4+5=9$ & $10.0 \%$ & & & & \\
\hline ADT & $80.0 \%$ & & & & \\
\hline Surgery & $10.0 \%$ & & & & \\
\hline Sleep Related Impairment (PROMIS SRI) & $42.5(10)$ & $43(10)$ & $44.6(10.2)$ & & \\
\hline Cognitive Fatigue (rPFS) & $1.95(1.7)$ & $2.6(2.3)$ & $1.7(1.6)$ & & \\
\hline
\end{tabular}

Two-sided Mann-Whitney $U$ test was used to evaluate group differences between EBRT and AS cohorts for PSA. Two-sided Independent $T$-test was used to evaluate group differences between EBRT and AS cohorts for normally distributed variables (age, BMI, RBC, and HB). Two-sided Wilcoxon signed-rank test was used to evaluate differences between EBRT-T1 and EBRT-T2 for PSA. Two-sided paired T-test was used to evaluate differences between EBRT-T1 and EBRT-T2 for normally distributed variables (RBC and $\mathrm{HB}$ ).

$B M I$ body mass index, $A D T$ androgen deprivation therapy, $H B$ hemoglobin, $R B C$ red blood cells, $P S A$ prostate-specific antigen, $F A C T-F$ the Functional Assessment Cancer Therapy-Fatigue, HAM-D Hamilton Depression Rating Scale, PROMIS patient-reported outcome measurement information system, SD sleep disturbances, SRI sleep-related impairment, rPFS revised Piper Fatigue Scale, EBRT external beam radiation therapy, AS active surveillance. ${ }^{*}$ Statistically significant.

other psychoneurological symptoms such as major depressive disorder [42] and anxiety in rheumatoid arthritis [43].

In comparison to the EBRT cohorts, in AS men, bivariate correlation analysis showed positive correlation between high concentrations of survivin and increased intensity of psychoneurological symptoms. Survivin is a member of an inhibitor of apoptosis family distinctly expressed in tumors [44]. We previously showed soluble survivin to be associated with cancer-related fatigue [22] in men with prostate cancer 3 months after radiation therapy. While published data support evidence for survivin leading to poor outcomes with cancer, to our knowledge, the present study is the first to examine survivin in relation to psychoneurological cancer-related symptoms.
In summary, we reported that both EBRT-T2 and AS clusters have the same set of symptoms closely interrelated within the cluster, but different immune biomarkers differentially correlated with each of these clusters. This distinction can be attributed to the differences in the clinical trajectories and clinical outcomes. We identified specific symptom clusters for each study group and distinct cytokines that were associated with each cluster. RANTES was the only chemokine that was significant in both EV-associated and soluble forms. In our previous report on men with prostate cancer before and after EBRT, we found more EV-associated cytokines were statistically significant correlated with worsening fatigue compared to soluble ones when looking at change in total concentration of cytokines over time [22]. Comparison of 


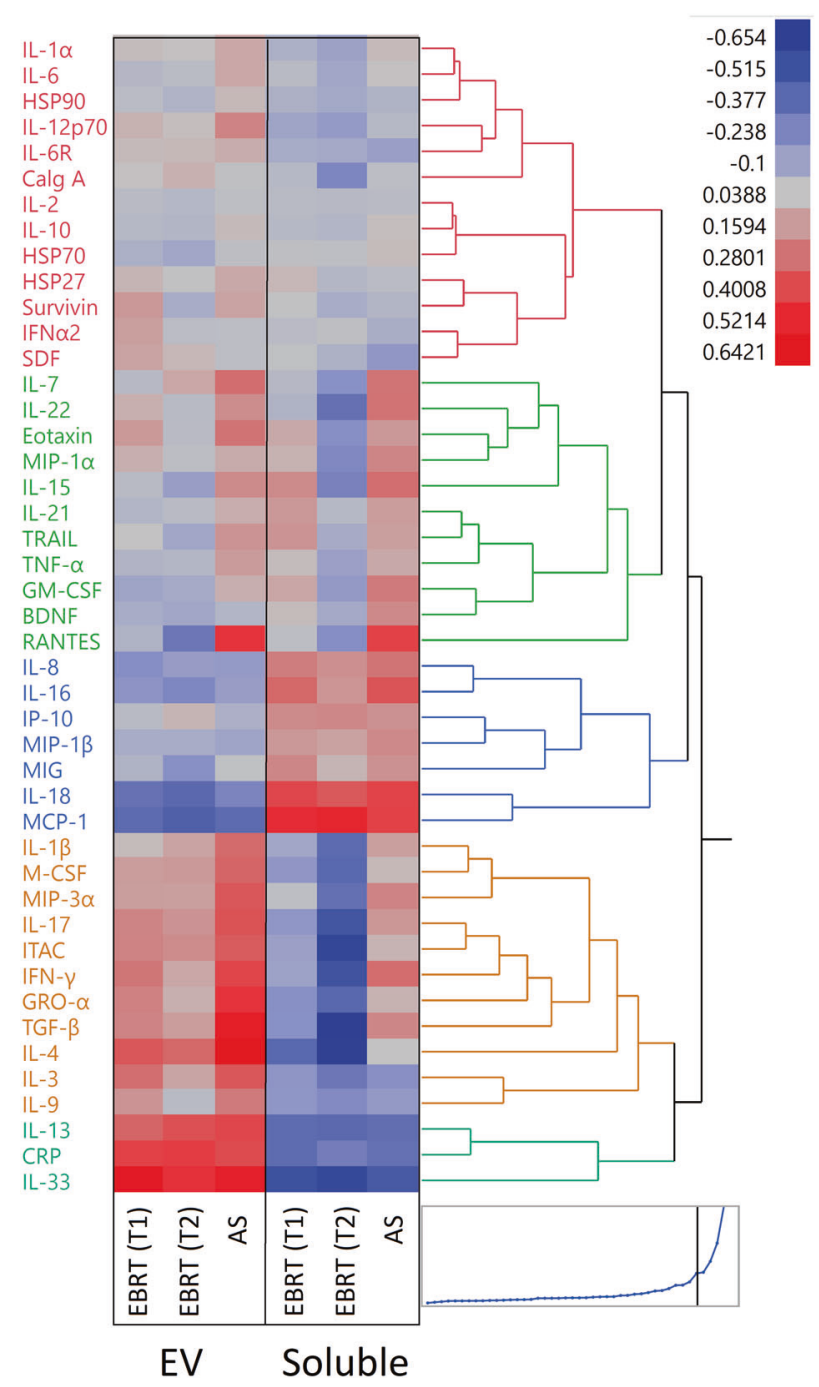

Fig. 3 Hierarchical cluster of average cytokines, chemokines, and heat-shock protein expression in logarithm base ten from multiplexed bead-based arrays. Clustering shows the relative differences in concentrations of the markers between EV and soluble fractions. The five different color-coded dendrograms shows clusters of biomarkers with similar expression values across the EV and soluble fractions. EBRT external beam radiation therapy, T1 at the start of radiation, T2 3 months post radiation, AS active surveillance.

EV-associated and soluble cytokines changes can be informative in discerning inflammatory pathways related to psychoneurological symptom clusters.

Our exploratory study has several limitations. First, the sample size was relatively small, thus preventing multiple testing correction. Second, blood collections were performed at different times of the day, which may introduce diurnal variation [45]. A third limitation of this study is that samples were collected from 2009 to 2014 and prolonged storage may have led to slow protein degradation. A study that evaluated stability of cytokines in plasma over 4 years concluded that IL-13, IL-15, IL-17, and CXCL8 showed degradation within one year, whereas IL-2, IL-4, $\mathrm{IL}-12$, and IL-18 were stable up to 3 years. Other cytokines (II-1a, IL-1B, IL-5, IL-6, and IL-10) had degradation up to $50 \%$ within $2-3$ years of storage [46]. There are no data in the literature regarding the stability of EVs over long-term storage. In the current study, both control and experimental samples were stored in the same way and were processed for EV extractions and multiplex assays
Table 2. Bivariate correlations between EBRT-T1, EBRT-T2, and AS symptom clusters and soluble and EV-associated immune markers.

\begin{tabular}{|c|c|c|}
\hline & Pearson $r$ & $P$ value \\
\hline \multicolumn{3}{|c|}{ Soluble cytokine correlations with EBRT-T1 cluster } \\
\hline RANTES & $0.380^{*}$ & 0.024 \\
\hline IL-21 & $-0.479^{* *}$ & 0.004 \\
\hline IL-33 & $-0.346^{*}$ & 0.042 \\
\hline \multicolumn{3}{|c|}{ EV-associated cytokine correlations with EBRT-T1 cluster } \\
\hline IL-1 $\alpha$ & $-0.375^{*}$ & 0.026 \\
\hline IL-15 & $-0.465^{* *}$ & 0.005 \\
\hline IL-21 & $-0.345^{*}$ & 0.042 \\
\hline GM-CSF & $-0.359^{*}$ & 0.034 \\
\hline M-CSF & $-0.335^{*}$ & 0.049 \\
\hline RANTES & $0.421^{*}$ & 0.012 \\
\hline \multicolumn{3}{|c|}{ Soluble cytokine correlations with EBRT-T2 cluster } \\
\hline IFN $\alpha 2$ & $0.413^{* *}$ & 0.009 \\
\hline IL-9 & $0.447^{* *}$ & 0.004 \\
\hline IL-17 & $0.371^{*}$ & 0.02 \\
\hline \multicolumn{3}{|c|}{ Soluble cytokine correlations with AS cluster } \\
\hline HSP90 & $-0.446^{*}$ & 0.049 \\
\hline Survivin & $0.474^{*}$ & 0.035 \\
\hline IL-1b & $-0.451^{*}$ & 0.046 \\
\hline \multicolumn{3}{|c|}{ EV-associated cytokine correlations with AS group } \\
\hline HSP70 & $-0.478^{*}$ & 0.033 \\
\hline MIG & $-0.548^{*}$ & 0.012 \\
\hline
\end{tabular}

EBRT external beam radiation therapy, $A S$ active surveillance, $T 1$ at the start of radiation.

${ }^{*} p<0.05,{ }^{* *} p<0.01$.

on the same day after defrosting samples to avoid repeated thaw freeze cycles. Lastly, we did not study which cell types the EVs in this study are most likely originating from and/or half-life of cytokines that are free vs. EV-associated. There are no published data regarding the possible difference between half-life of free and EV-associated cytokines. In general, cytokines are relatively stable compounds [46]. A recent study examining stability of EV proteins, RNA, and other EV markers at RT, $4^{\circ} \mathrm{C}$, and $-70^{\circ} \mathrm{C}$ found that CD63 and hsp70found that faster degradation of CD63 and HSP70 was reached when incubated at RT and $4{ }^{\circ} \mathrm{C}$ compared to 10 days at $-70^{\circ} \mathrm{C}$ [47]. We stored our samples at $-80^{\circ} \mathrm{C}$. Because most of clinical research takes years for initial data collection, future studies with longer storage (5-10 years) should investigate the effect of degradation and half-life of cytokines in soluble form and EV associated. In relation to cell-specific origin of EVs, it is plausible that some EV-associated cytokines are originating from prostate cancer cells, muscle cells, vascular origin, or neuronal origin. Numerous studies have recently shown that EVs may cross the blood-brain barrier and neuronal EV-associated biomarkers have been detected in circulating plasma using an immunoaffinity assay (e.g. EV + CD171) [11, 48].

Nevertheless, these findings are of important clinical relevance as they suggest that cancer and cancer treatments may drive cooccurrence of symptoms resulting in specific symptom clusters. The cytokines that positively correlated with worsening symptoms have known pro- inflammatory effects, which supports our observation that inflammation is associated with a worsening psychoneurological state in patients with prostate cancer. This observation supports the notion that worsening symptoms may be affected by the inflammatory processes and changes in these processes (worsening or improvement of inflammation) have a 
consequential effect on the symptom clusters. The knowledge that EV-associated cytokines significantly correlate with worsening symptoms may unravel distinct pathways. Future studies will reveal the roles of specific EVs, mainly, neuronally derived ones and their association of cytokines to separate the impact of central vs. peripheral inflammation.

\section{DATA AVAILABILITY}

The data that support the findings of this study are available on reasonable request to the corresponding author.

\section{REFERENCES}

1. Reilly CM, Bruner DW, Mitchell SA, Minasian LM, Basch E, Dueck AC, et al. A literature synthesis of symptom prevalence and severity in persons receiving active cancer treatment. Supportive Care Cancer. 2013;21:1525-50.

2. Miaskowski C, Barsevick A, Berger A, Casagrande R, Grady P, Jacobsen P. Advancing symptom science through symptom cluster research. J Natl Cancer Inst. 2017;109:1-9.

3. Bower JE. Cancer-related fatigue-mechanisms, risk factors, and treatments. Nat Rev Clin Oncol. 2014;11:597-609.

4. Kim SH, Yoon SM, Choi YD, Choi E, Song H. Predictors of health-related quality of life in Korean prostate cancer patients receiving androgen deprivation therapy. Eur J Oncol Nurs. 2017;30:84-90.

5. Kim H-J, Barsevick AM, Fang CY, Miaskowski C. Common biological pathways underlying the psychoneurological symptom cluster in cancer patients. Cancer Nurs. 2012;35:E1-E20.

6. Dantzer R, O'Connor JC, Freund GG, Johnson RW, Kelley KW. From inflammation to sickness and depression: when the immune system subjugates the brain. Nat Rev Neurosci. 2008;9:46-56.

7. Dantzer R. Cytokine, sickness behavior, and depression. Immunol Allergy Clin. 2009;29:247-64.

8. Capuron L, Ravaud A, Miller AH, Dantzer R. Baseline mood and psychosocial characteristics of patients developing depressive symptoms during interleukin-2 and/or interferon-alpha cancer therapy. Brain Behav Immun. 2004;18:205-13.

9. Umekawa K, Kimura T, Kudoh S, Suzumura T, Oka T, Nagata $M$, et al. Plasma RANTES, IL-10, and IL-8 levels in non-small-cell lung cancer patients treated with EGFR-TKIs. BMC Res Notes. 2013;6:139.

10. Tyring S, Gottlieb A, Papp K, Gordon K, Leonardi C, Wang A, et al. Etanercept and clinical outcomes, fatigue, and depression in psoriasis: double-blind placebocontrolled randomised phase III trial. Lancet. 2006;367:29-35.

11. Guedes VA, Devoto C, Leete J, Sass D, Acott DJ, Mithani S, et al. Extracellular vesicle proteins and microRNAs as biomarkers for traumatic brain injury. Front Neurol. 2020;11:1-18.

12. Kalluri R. The biology and function of exosomes in cancer. J Clin Invest 2016;126:1208-15.

13. Théry C, Witwer KW, Aikawa E, Alcaraz MJ, Anderson JD, Andriantsitohaina $R$ et al. Minimal information for studies of extracellular vesicles 2018 (MISEV2018): a position statement of the International Society for Extracellular Vesicles and update of the MISEV2014 guidelines. J Extracell Vesicles. 2018;7:1535750.

14. Fitzgerald W, Freeman $M L$, Lederman $M M$, Vasilieva $E$, Romero R, Margolis L. A system of cytokines encapsulated in extracellular vesicles. Sci Rep. 2018;8:1-11.

15. Yellen SB, Cella DF, Webster K, Blendowski C, Kaplan E. Measuring fatigue and other anemia-related symptoms with the Functional Assessment of Cancer Therapy (FACT) measurement system. J Pain Symptom Manage. 1997; 13:63-74

16. Cella D, Eton DT, Lai J-S, Peterman AH, Merkel DE. Combining anchor and distribution-based methods to derive minimal clinically important differences on the Functional Assessment of Cancer Therapy (FACT) anemia and fatigue scales. J Pain Symptom Manage. 2002;24:547-61.

17. Bagby RM, Ryder AG, Schuller DR, Marshall MB. The Hamilton Depression Rating Scale: has the gold standard become a lead weight? Am J Psychiatry. 2004;161:2163-77.

18. Zimmerman M, Martinez JH, Young D, Chelminski I, Dalrymple K. Severity classification on the Hamilton depression rating scale. J Affect Disord. 2013;150:384-8.

19. Yu L, Buysse DJ, Germain A, Moul DE, Stover A, Dodds NE, et al. Development of short forms from the PROMIS ${ }^{\mathrm{TM}}$ sleep disturbance and sleep-related impairment item banks. Behav Sleep Med. 2012;10:6-24.

20. Buysse DJ, Yu L, Moul DE, Germain A, Stover A, Dodds NE, et al. Development and validation of patient-reported outcome measures for sleep disturbance and sleep-related impairments. Sleep. 2010;33:781-92.
21. Piper BF, Dibble SL, Dodd MJ, Weiss MC, Slaughter RE, Paul SM. The revised Piper Fatigue Scale: psychometric evaluation in women with breast cancer. Oncol Nurs Forum. 1998;25:677-684.

22. Sass D, Fitzgerald W, Barb JJ, Kupzyk K, Margolis L, Saligan L. An exploratory analysis of extracellular vesicle-associated and soluble cytokines in cancerrelated fatigue in men with prostate cancer. Brain Behav Immun Health. 2020;9:100140

23. Jackson DA. Stopping rules in principal components analysis: a comparison of heuristical and statistical approaches. Ecology. 1993;74:2204-14.

24. Dziuban CD, Shirkey EC. When is a correlation matrix appropriate for factor analysis? Some decision rules. Psychol bull. 1974;81:358-361.

25. Kim H-J, McGuire DB, Tulman L, Barsevick AM. Symptom clusters: concept analysis and clinical implications for cancer nursing. Cancer Nurs. 2005;28:270-82.

26. Feng LR, Wolff BS, Lukkahatai N, Espina A, Saligan LN. Exploratory investigation of early biomarkers for chronic fatigue in prostate cancer patients following radiation therapy. Cancer Nurs. 2017;40:184-93.

27. Sotelo JL, Musselman D, Nemeroff C. The biology of depression in cancer and the relationship between depression and cancer progression. Int Rev Psychiatry. 2014:26:16-30.

28. Cheung YT, Ng T, Shwe M, Ho HK, Foo KM, Cham MT, et al. Association of proinflammatory cytokines and chemotherapy-associated cognitive impairment in breast cancer patients: a multi-centered, prospective, cohort study. Ann Oncol. 2015;26:1446-51.

29. Appay V, Rowland-Jones SL. RANTES: a versatile and controversial chemokine. Trends Immunol. 2001;22:83-7.

30. Ogłodek EA, Szota A, Just MJ, Moś D, Araszkiewicz A. Comparison of chemokines (CCL-5 and SDF-1), chemokine receptors (CCR-5 and CXCR-4) and IL-6 levels in patients with different severities of depression. Pharmacol Rep. 2014;66:920-6.

31. Yang $\mathrm{CH}$, Murti A, Pfeffer SR, Basu L, Kim JG, Pfeffer LM. IFNa/ $\beta$ promotes cell survival by activating NF-KB. Proc Natl Acad Sci USA. 2000;97:13631-6.

32. Gaffen SL. Structure and signalling in the IL-17 receptor family. Nat Rev Immunol. 2009;9:556-67.

33. Malik S, Awasthi A. Transcriptional control of Th9 cells: Role of foxo1 in interleukin-9 induction. Front Immunol. 2018;9:995.

34. Bower JE, Ganz PA, Irwin MR, Arevalo JM, Cole SW. Fatigue and gene expression in human leukocytes: increased NF-KB and decreased glucocorticoid signaling in breast cancer survivors with persistent fatigue. Brain Behav Immun. 2011;25:147-50.

35. Irwin MR, Wang M, Ribeiro D, Cho HJ, Olmstead R, Breen EC, et al. Sleep loss activates cellular inflammatory signaling. Biol Psychiatry. 2008;64:538-40.

36. Elovainio M, Taipale T, Seppälä I, Mononen N, Raitoharju E, Jokela M, et al Activated immune-inflammatory pathways are associated with long-standing depressive symptoms: evidence from gene-set enrichment analyses in the Young Finns Study. J Psychiatr Res. 2015;71:120-5.

37. Capuron L, Gumnick JF, Musselman DL, Lawson DH, Reemsnyder A, Nemeroff CB, et al. Neurobehavioral effects of interferon-a in cancer patients: phenomenology and paroxetine responsiveness of symptom dimensions. Neuropsychopharmacology. 2002;26:643-52.

38. Cooper CM, Godlewska B, Sharpley AL, Barnes E, Cowen PJ, Harmer CJ. Interferona induces negative biases in emotional processing in patients with hepatitis $C$ virus infection: a preliminary study. Psychol Med. 2018;48:998-1007.

39. Nowak EC, Weaver CT, Turner H, Begum-Haque S, Becher B, Schreiner B, et al. IL-9 as a mediator of Th17-driven inflammatory disease. J Exp Med. 2009;206:1653-60.

40. Hamre H, Zeller B, Kanellopoulos A, Ruud E, Fosså SD, Loge JH, et al. Serum cytokines and chronic fatigue in adults surviving after childhood leukemia and lymphoma. Brain Behav Immun. 2013;30:80-7.

41. McGeachy MJ, Cua DJ, Gaffen SL. The IL-17 family of cytokines in health and disease. Immunity. 2019;50:892-906.

42. Davami MH, Baharlou R, Ahmadi Vasmehjani A, Ghanizadeh A, Keshtkar M Dezhkam I, et al. Elevated IL-17 and TGF- $\beta$ serum levels: a positive correlation between T-helper 17 cell-related pro-inflammatory responses with major depressive disorder. Basic Clin Neurosci. 2016;7:137-42.

43. Liu Y, HO RCM, Mak A. The role of interleukin (IL)-17 in anxiety and depression of patients with rheumatoid arthritis. Int J Rheum Dis. 2012;15:183-7.

44. Jaiswal PK, Goel A, Mittal R. Survivin: a molecular biomarker in cancer. Indian J Med Res. 2015;141:389-97.

45. Witwer KW, Buzás El, Bemis LT, Bora A, Lässer C, Lötvall J, et al. Standardization of sample collection, isolation and analysis methods in extracellular vesicle research. J Extracell Vesicles. 2013;2:20360.

46. de Jager W, Bourcier K, Rijkers GT, Prakken BJ, Seyfert-Margolis V. Prerequisites for cytokine measurements in clinical trials with multiplex immunoassays. BMC Immunol. 2009;10:1-11.

47. Lee M, Ban J-J, Im W, Kim M. Influence of storage condition on exosome recovery. Biotechnol Bioprocess Eng. 2016;21:299-304. 
48. Mustapic M, Eitan E, Werner JK Jr, Berkowitz ST, Lazaropoulos MP, Tran J, et al. Plasma extracellular vesicles enriched for neuronal origin: a potential window into brain pathologic processes. Front Neurosci. 2017;11:278.

\section{ACKNOWLEDGEMENTS}

This work is supported by the National Institute of Nursing Research and the Section of Intercellular Communication in the National Institute of Child Health and Human Development, National Institutes of Health. The authors thank Dera Tompkins, NIH Library Writing Center, for manuscript editing assistance.

\section{AUTHOR CONTRIBUTIONS}

Original idea, planning, and input: D.S., L.S., L.M., and A.M.B.; supervision of the project: L.S. and L.M.; resources: L.S. and L.M.; original experimental work: D.S. and W.F.; data analysis: D.S., K.K., and J.J.B.; data curation: D.S. and L.S.; original drafting of manuscript: D.S., L.S., L.M., and I.T.; review and editing: D.S., L.S., W.F., A.M.B., I.T., J.J.B., K.K., and L.M.

\section{FUNDING}

Open Access funding provided by the National Institutes of Health (NIH).

\section{COMPETING INTERESTS}

The authors declare no competing interests.

\section{ADDITIONAL INFORMATION}

Supplementary information The online version contains supplementary material available at https://doi.org/10.1038/s41398-021-01554-w.

Correspondence and requests for materials should be addressed to L.S.

Reprints and permission information is available at http://www.nature.com/ reprints

Publisher's note Springer Nature remains neutral with regard to jurisdictional claims in published maps and institutional affiliations.

Open Access This article is licensed under a Creative Commons Attribution 4.0 International License, which permits use, sharing, adaptation, distribution and reproduction in any medium or format, as long as you give appropriate credit to the original author(s) and the source, provide a link to the Creative Commons license, and indicate if changes were made. The images or other third party material in this article are included in the article's Creative Commons license, unless indicated otherwise in a credit line to the material. If material is not included in the article's Creative Commons license and your intended use is not permitted by statutory regulation or exceeds the permitted use, you will need to obtain permission directly from the copyright holder. To view a copy of this license, visit http://creativecommons. org/licenses/by/4.0/.

This is a U.S. government work and not under copyright protection in the U.S.; foreign copyright protection may apply 2021 